\title{
Availability and Profit Analysis of a linear Consecutive 2-out-of-4 Repairable System with Units Exchange
}

\author{
U.A. Ali ${ }^{1}$, Ibrahim Yusuf, ${ }^{2, *}$ \\ ${ }^{1}$ Department of Mathematics, Usmanu Danfodio University, Sokoto, Nigeria \\ ${ }^{2}$ Department of Mathematical Sciences, Faculty of Science, Bayero University, Kano, Nigeria \\ *Corresponding author: Ibrahimyusuffagge@gmail.com
}

Received September 16, 2013; Revised December 16, 2013; Accepted March 13, 2014

\begin{abstract}
In this paper, we study some reliability characteristics of a repairable linear consecutive 2-out-of-4 system. The system work when to two units in a row (consecutive) works. The system is attended by three repairmen. When an operating unit failed, a standby is switched on or an idle operating unit is exchange with the failed unit. The explicit expressions of the reliability characteristics such availability, busy period of the repairmen and profit function are derived. Some cases are analyzed graphically to investigate the impact of system parameters on availability and profit.
\end{abstract}

Keywords: availability, profit, repairable system

Cite This Article: U.A. Ali, and Ibrahim Yusuf, "Availability and Profit Analysis of a linear Consecutive 2out-of-4 Repairable System with Units Exchange.” American Journal of Applied Mathematics and Statistics, vol. 2, no. 2 (2014): 83-87. doi: 10.12691/ajams-2-2-6.

\section{Introduction}

The redundancy issues in systems play the key role for a successful design and functionality of sophisticated systems. One of the forms of redundancy is the k-out-of-n system which has wide application in industrial setting, nuclear power plants, computer systems, automobiles etc.. There are redundant systems of three/four units in which two/three units are sufficient to perform the entire function of the system. Examples of such systems are 2-out-of-3, 2out-of-4, or 3-out-of-4 redundant systems. Availability and profit of redundant system may be enhancing using highly reliable structural design of the system or subsystem of higher reliability. Improving the reliability and availability of system/subsystem, the production and associated profit will also increase. Increase in production lead to the increase of profit. This can be achieve be maintaining reliability and availability at highest order. To achieve high production and profit, the system should remain operative (available) for maximum possible duration. [1] studied the availability of k-out-of-n G system with non identical components subject to repair priorities. [2] studied the availability and profit of 3-outof-4 repairable system.

Due to their importance in industries and design, redundant systems have received attention from different researchers (see, for instance, [5]). Reliability evaluation play a key role in engineering design and have been effectively applied to enhance performance (see for instance $[3,4,7])$. [9] analyzed an r-out-of-n system with several repairmen.
In this paper, we study some reliability characteristics of a repairable linear consecutive 2-out-of-4 system to determine how availability and profit function can be improved by exchanging the failed unit with an idle unit.

The contribution of this paper is twofold. First, is to develop the explicit expressions for steady state availability, busy period and profit function. The second is to perform a parametric investigation of system parameters on system availability and profit function and capture their effect on availability and profit function. The rest of the paper is organized as follows. Section 2 gives the notations, transition diagram and states of the system. Section 3 presents materials and methods of the study. Section 4 presents derivation of reliability models. The results of our numerical simulations are presented and discussed in Section 5. The paper is concluded in Section 6.

\section{Notations, Transition Diagram and States of the System}

\section{States of the System}

Up States

$$
\begin{aligned}
& S_{0}\left(A_{1 O}, A_{2 O}, A_{3 S}, A_{4 S}\right), S_{1}\left(A_{1 R}, A_{2 O}, A_{3 O}, A_{4 S}\right), \\
& S_{2}\left(A_{1 L}, A_{2 R}, A_{3 O}, A_{4 O}\right), S_{3}\left(A_{1 R}, A_{2 R}, A_{3 O}, A_{4 O}\right), \\
& S_{6}\left(A_{1 R}, A_{3 R}, A_{1 O}, A_{4 O}\right)
\end{aligned}
$$

Down States

$$
S_{4}\left(A_{1 L}, A_{2 R}, A_{3 R}, A_{4 L}\right), S_{5}\left(A_{1 R}, A_{2 R}, A_{3 R}, A_{4 L}\right)
$$

Notations 
$\alpha_{i}$ : Repair rate of unit $A_{i}, i=1,2,3$;

$\beta_{i}$ : Failure rate of unit $A_{i}$;

$\mu$ : Exchange rate;

$A_{q}:$ Steady state availability;

$B_{V}$ : Busy period;

$P F$ : Profit function;

$A_{10}$ : Unit in operation;

$A_{i S}:$ Unit in standby;

$A_{i R}:$ Unit under repair;

$A_{i L}$ : Idle unit.

\section{Materials and Methods}

In this study, reliability analysis of the system is analyzed by making use of Kolmogorov forward equations method and expressions for various measures of system effectiveness such as steady state availability, busy period of repairmen and profit incurred to the system.

\section{Reliability Analysis of the System}

Let $P(t)$ be the probability row vector and $P_{i}(t)$ be the probability that the system is in the states $S_{i}$ at time $t \geq 0$

The initial conditions for this problem are as :

$$
\begin{aligned}
& P(0)=\left[P_{0}(0), P_{1}(0), P_{2}(0), P_{3}(0), P_{4}(0), P_{5}(0), P_{6}(0)\right] \\
& =[1,0,0,0,0,0,0]
\end{aligned}
$$

we obtain the following system of differential equations from Figure 1:

$$
\begin{aligned}
& P_{0}^{\prime}(t)=y_{1} P_{0}(t)+\alpha_{1} P_{1}(t)+\alpha_{2} P_{2}(t) \\
& P_{1}^{\prime}(t)=y_{2} P_{2}(t)+\beta_{1} P_{0}(t)+\alpha_{2} P_{3}(t) \\
& P_{2}^{\prime}(t)=y_{3} P_{2}(t)+\beta_{2} P_{0}(t)+\alpha_{1} P_{2}(t)+\alpha_{3} P_{4}(t) \\
& P_{3}^{\prime}(t)=y_{4} P_{3}(t)+\beta_{2} P_{1}(t)+\alpha_{3} P_{5}(t) \\
& P_{4}^{\prime}(t)=y_{5} P_{4}(t)+\beta_{3} P_{2}(t) \\
& P_{5}^{\prime}(t)=y_{6} P_{5}(t)+\beta_{3} P_{3}(t)+\beta_{1} P_{6}(t) \\
& P_{6}^{\prime}(t)=y_{7} P_{6}(t)+\mu P_{4}(t)+\alpha_{1} P_{5}(t) \\
& P_{1}^{\prime}(t)=y_{2} P_{2}(t)+\beta_{1} P_{0}(t)+\alpha_{2} P_{3}(t)
\end{aligned}
$$

where

$$
\begin{aligned}
& y_{1}=-\left(\beta_{1}+\beta_{2}\right), y_{2}=-\left(\alpha_{1}+\beta_{2}\right), \\
& y_{3}=-\left(\alpha_{2}+\beta_{3}\right), y_{4}=-\left(\alpha_{1}+\alpha_{2}+\beta_{3}\right), \\
& y_{5}=-\left(\alpha_{3}+\mu\right), y_{6}=-\left(\alpha_{1}+\alpha_{3}\right), \\
& y_{7}=-\beta_{1}
\end{aligned}
$$

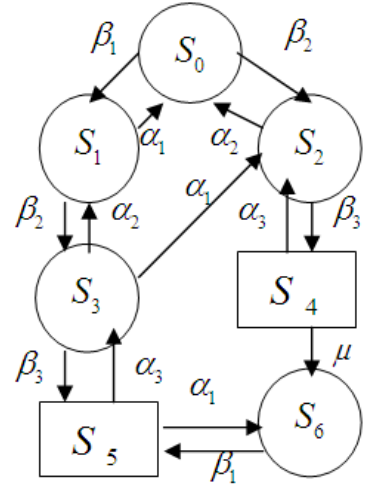

Figure 1. Transition diagram of the system

\subsection{Availability and Busy period Analysis}

The differential equations in (1) above is transformed into matrix as

$$
P^{\prime}=T P
$$

where

$$
T=\left[\begin{array}{ccccccc}
y_{1} & \alpha_{1} & \alpha_{2} & 0 & 0 & 0 & 0 \\
\beta_{1} & y_{2} & 0 & \alpha_{2} & 0 & 0 & 0 \\
\beta_{2} & 0 & y_{3} & \alpha_{1} & \alpha_{3} & 0 & 0 \\
0 & \beta_{2} & 0 & y_{4} & 0 & \alpha_{3} & 0 \\
0 & 0 & \beta_{3} & 0 & y_{5} & 0 & 0 \\
0 & 0 & 0 & \beta_{3} & 0 & y_{6} & \beta_{1} \\
0 & 0 & 0 & 0 & \mu & \alpha_{1} & y_{7}
\end{array}\right]
$$

The system of differential equations in (1) for the system above can be expressed in matrix form as:

$\left[\begin{array}{c}P_{0}^{\prime}(t) \\ P_{1}^{\prime}(t) \\ P_{2}^{\prime}(t) \\ P_{3}^{\prime}(t) \\ P_{4}^{\prime}(t) \\ P_{5}^{\prime}(t) \\ P_{6}^{\prime}(t)\end{array}\right]=\left[\begin{array}{ccccccc}y_{1} & \alpha_{1} & \alpha_{2} & 0 & 0 & 0 & 0 \\ \beta_{1} & y_{2} & 0 & \alpha_{2} & 0 & 0 & 0 \\ \beta_{2} & 0 & y_{3} & \alpha_{1} & \alpha_{3} & 0 & 0 \\ 0 & \beta_{2} & 0 & y_{4} & 0 & \alpha_{3} & 0 \\ 0 & 0 & \beta_{3} & 0 & y_{5} & 0 & 0 \\ 0 & 0 & 0 & \beta_{3} & 0 & y_{6} & \beta_{1} \\ 0 & 0 & 0 & 0 & \mu & \alpha_{1} & y_{7}\end{array}\right] \cdot\left[\begin{array}{c}P_{0}(t) \\ P_{1}(t) \\ P_{2}(t) \\ P_{3}(t) \\ P_{4}(t) \\ P_{5}(t) \\ P_{6}(t)\end{array}\right]$

Let $V$ be the time to failure of the system for system. Following [8], the steady-state availability and busy Period are given by

$$
A_{v}(\infty)=P_{0}(\infty)+P_{1}(\infty)+P_{2}(\infty)+P_{3}(\infty)+P_{6}(\infty)
$$

$$
B_{v}(\infty)=P_{1}(\infty)+P_{2}(\infty)+P_{3}(\infty)+P_{4}(\infty)+P_{5}(\infty)+P_{6}(\infty)
$$

In steady state, the derivatives of state probabilities become zero, thus (2) becomes

$$
T P=0
$$

which is in matrix form as 


$$
\left[\begin{array}{ccccccc}
y_{1} & \alpha_{1} & \alpha_{2} & 0 & 0 & 0 & 0 \\
\beta_{1} & y_{2} & 0 & \alpha_{2} & 0 & 0 & 0 \\
\beta_{2} & 0 & y_{3} & \alpha_{1} & \alpha_{3} & 0 & 0 \\
0 & \beta_{2} & 0 & y_{4} & 0 & \alpha_{3} & 0 \\
0 & 0 & \beta_{3} & 0 & y_{5} & 0 & 0 \\
0 & 0 & 0 & \beta_{3} & 0 & y_{6} & \beta_{1} \\
0 & 0 & 0 & 0 & \mu & \alpha_{1} & y_{7}
\end{array}\right]\left[\begin{array}{l}
P_{0}(t) \\
P_{1}(t) \\
P_{2}(t) \\
P_{3}(t) \\
P_{4}(t) \\
P_{5}(t) \\
P_{6}(t)
\end{array}\right]=\left[\begin{array}{l}
0 \\
0 \\
0 \\
0 \\
0 \\
0 \\
0
\end{array}\right]
$$

Using the following normalizing condition

$$
\sum_{i=0}^{6} P_{i}(\infty)=1
$$

We solve the system of linear equations in matrix above using (7) to obtain the state probabilities $P_{0}(\infty), P_{1}(\infty), P_{2}(\infty), \ldots, P_{6}(\infty)$

Expression for steady-state availability and busy period are thus:

$$
A_{v}(\infty)=\frac{N_{1}}{D_{1}}, \quad B_{v}(\infty)=\frac{N_{2}}{D_{1}}
$$

Where

$$
\begin{aligned}
& N_{1}=\alpha_{1} \alpha_{2} \alpha_{3} \beta_{1}\left(\alpha_{1} \mu+\alpha_{2} \mu+\beta_{3} \mu+\beta_{2} \mu+\alpha_{1} \alpha_{3}+\alpha_{2} \alpha_{3}+\alpha_{3} \beta_{2}\right) \\
& \left.+\alpha_{2} \alpha_{3} \beta_{1} \alpha_{1} \beta_{1} \mu+\alpha_{2} \beta_{1} \mu+\beta_{2} \beta_{3} \mu+\beta_{1} \beta_{3} \mu+\alpha_{1} \alpha_{3} \beta_{1}+\alpha_{2} \alpha_{3} \beta_{1}\right) \\
& +\alpha_{1} \alpha_{3} \beta_{1} \beta_{2}\left(\alpha_{1} \mu+\alpha_{2} \mu+\beta_{1} \mu+\beta_{2} \mu+\alpha_{1} \alpha_{3}+\alpha_{2} \alpha_{3}+\alpha_{3} \beta_{2}\right. \\
& \left.+\alpha_{3} \beta_{1}\right)+\alpha_{3} \beta_{1} \beta_{2}\left(\alpha_{1} \beta_{3} \mu+\alpha_{2} \beta_{1} \mu+\beta_{2} \beta_{3} \mu+\beta_{1} \beta_{3} \mu+\alpha_{2} \alpha_{3} \beta_{1}\right) \\
& +\alpha_{1} \beta_{2} \beta_{3}\left(\beta_{1} \beta_{3} \mu+\alpha_{3} \beta_{1} \mu+\alpha_{2} \beta_{1} \mu+\alpha_{1} \beta_{1} \mu+\beta_{2} \beta_{3} \mu+\alpha_{1} \beta_{2} \mu\right. \\
& \left.+\alpha_{3} \beta_{2} \mu+\alpha_{1} \beta_{3} \mu+\alpha_{1} \alpha_{3} \mu+\alpha_{2} \alpha_{3} \mu+\alpha_{1} \alpha_{2} \mu+\alpha_{2} \alpha_{3} \beta_{1}+\alpha_{1}^{2} \mu\right) \\
& N_{2}=\alpha_{2} \alpha_{3} \beta_{1}\left(\alpha_{1} \beta_{1} \mu+\alpha_{2} \beta_{1} \mu+\beta_{2} \beta_{3} \mu+\beta_{1} \beta_{3} \mu+\alpha_{1} \alpha_{3} \beta_{1}+\alpha_{2} \alpha_{3} \beta_{1}\right) \\
& +\alpha_{1} \alpha_{3} \beta_{1} \beta_{2}\left(\alpha_{1} \mu+\alpha_{2} \mu+\beta_{1} \mu+\beta_{2} \mu+\alpha_{1} \alpha_{3}+\alpha_{2} \alpha_{3}+\alpha_{3} \beta_{2}+\alpha_{3} \beta_{1}\right) \\
& +\alpha_{3} \beta_{2} \beta_{1}\left(\alpha_{1} \beta_{3} \mu+\alpha_{2} \beta_{1} \mu+\beta_{2} \beta_{3} \mu+\beta_{1} \beta_{3} \mu+\alpha_{2} \alpha_{3} \beta_{1}\right) \\
& +\alpha_{1} \alpha_{3} \beta_{1} \beta_{2} \beta_{3}\left(\alpha_{1}+\alpha_{2}+\beta_{1}+\beta_{2}\right)+\beta_{1} \beta_{2} \beta_{3}\left(\alpha_{1}^{2} \mu+\alpha_{1} \alpha_{2} \mu+\alpha_{1} \beta_{2} \mu\right. \\
& \left.+\alpha_{1} \beta_{1} \mu+\alpha_{1} \beta_{3} \mu+\alpha_{2} \beta_{1} \mu+\beta_{1} \beta_{3} \mu+\beta_{2} \beta_{3} \mu+\alpha_{2} \alpha_{3} \beta_{1}\right) \\
& +\alpha_{1} \beta_{2} \beta_{3}\left(\beta_{1} \beta_{3} \mu+\alpha_{3} \beta_{1} \mu+\alpha_{2} \beta_{1} \mu+\alpha_{1} \beta_{1} \mu+\beta_{2} \beta_{3} \mu+\alpha_{1} \beta_{2} \mu\right. \\
& \left.+\alpha_{3} \beta_{2} \mu+\alpha_{1} \beta_{3} \mu+\alpha_{1} \alpha_{3} \mu+\alpha_{2} \alpha_{3} \mu+\alpha_{1} \alpha_{2} \mu+\alpha_{1}^{2} \mu+\alpha_{2} \alpha_{3} \beta_{1}\right) \\
& D_{1}=\alpha_{2}^{2} \alpha_{3}^{2} \beta_{1}^{2}+\alpha_{2} \alpha_{3}^{2} \beta_{1}^{2} \beta_{2}+\alpha_{1} \alpha_{3} \beta_{1} \beta_{2}^{2} \beta_{3}+\alpha_{1}^{2} \alpha_{3} \beta_{1} \beta_{2} \beta_{3} \\
& +2 \alpha_{1} \alpha_{2} \alpha_{3} \beta_{1} \beta_{2} \beta_{3}+2 \alpha_{1} \alpha_{2} \alpha_{3}^{2} \beta_{1} \beta_{2}+\alpha_{1}^{2} \alpha_{2} \beta_{2} \beta_{3} \mu \\
& +\alpha_{1}^{2} \alpha_{3} \beta_{1} \beta_{2} \mu+\alpha_{1} \alpha_{2} \alpha_{3} \beta_{1} \beta_{3} \mu+\alpha_{2} \alpha_{3} \beta_{1} \beta_{2} \beta_{3} \mu+\alpha_{2} \alpha_{3} \beta_{1}^{2} \beta_{2} \beta_{3} \\
& +\alpha_{1} \alpha_{3} \beta_{1}^{2} \beta_{2} \beta_{3}+\alpha_{1} \alpha_{3} \beta_{1}^{2} \beta_{2} \mu+\alpha_{2} \alpha_{3} \beta_{1}^{2} \beta_{2} \mu+2 \alpha_{1} \alpha_{2} \beta_{1} \beta_{2} \beta_{3} \mu \\
& +\alpha_{3} \beta_{1}^{2} \beta_{2} \beta_{3} \mu+\alpha_{1} \alpha_{2} \alpha_{3} \beta_{2} \beta_{3} \mu+2 \alpha_{1} \alpha_{3} \beta_{1} \beta_{2} \beta_{3} \mu \\
& +2 \alpha_{1} \alpha_{2} \alpha_{3} \beta_{1} \beta_{2} \mu+\alpha_{1} \alpha_{2} \alpha_{3} \beta_{1}^{2} \mu+\alpha_{2} \alpha_{3} \beta_{1}^{2} \beta_{3} \mu+\alpha_{3} \beta_{1} \beta_{2}^{2} \beta_{3} \mu \\
& +\alpha_{1} \beta_{1} \beta_{2}^{2} \beta_{3} \mu+\alpha_{1} \alpha_{2} \alpha_{3}^{2} \beta_{1}^{2}+\alpha_{1} \alpha_{3}^{2} \beta_{1}^{2} \beta_{2}+\alpha_{1} \alpha_{3}^{2} \beta_{1} \beta_{2}^{2}+\alpha_{1}^{2} \alpha_{3}^{2} \beta_{1} \beta_{2} \\
& +\alpha_{1} \alpha_{2}^{2} \alpha_{3}^{2} \beta_{1}+\alpha_{1}^{2} \alpha_{2} \alpha_{3}^{2} \beta_{1}+\alpha_{1} \beta_{2}^{2} \beta_{3}^{2} \mu+\alpha_{1}^{2} \beta_{2} \beta_{3}^{2} \mu+\alpha_{1} \alpha_{3} \beta_{1} \beta_{2}^{2} \mu \\
& +\alpha_{1} \beta_{1}^{2} \beta_{2} \beta_{3} \mu+\alpha_{2}^{2} \alpha_{3} \beta_{1}^{2} \mu+\alpha_{1}^{2} \beta_{3} \beta_{2}^{2} \mu+\beta_{1} \beta_{2}^{2} \beta_{3}^{2} \mu+\beta_{1}^{2} \beta_{2} \beta_{3}^{2} \mu \\
& +\alpha_{1}^{3} \beta_{2} \beta_{3} \mu+\alpha_{1} \alpha_{3} \beta_{2}^{2} \beta_{3} \mu+\alpha_{1}^{2} \alpha_{3} \beta_{2} \beta_{3}+2 \alpha_{1}^{2} \beta_{1} \beta_{2} \beta_{3} \mu \\
& +\alpha_{2} \beta_{1}^{2} \beta_{2} \beta_{3} \mu+2 \alpha_{1} \beta_{1} \beta_{2} \beta_{3}^{2} \mu+\alpha_{1}^{2} \alpha_{2} \alpha_{3} \beta_{1} \mu+\alpha_{1} \alpha_{2}^{2} \alpha_{3} \beta_{1} \mu
\end{aligned}
$$

\subsection{Profit Analysis}

The units and supporting unit are subjected to corrective maintenance in states 2, 3, 5 and 6 and 4 respectively. Let $C_{0}$ and $C_{1}$ be the revenue generated when the system is in working state and no income when in failed state, and accumulated cost of each repair for failed units (corrective maintenance) respectively. Following $[6,8]$ the expected total profit per unit time incurred to the system in the steady-state is

$$
P F=C_{0} A_{V}(\infty)-C_{1} B_{V}(\infty)
$$

\subsection{Special Case}

When no unit exchange is allowed, the steady-state availability and period, and profit function are given by:

$$
\begin{gathered}
A_{v}^{*}(\infty)=P_{0}(\infty)+P_{1}(\infty)+P_{2}(\infty)+P_{3}(\infty)=\frac{N_{3}}{D_{2}} \\
B_{V}^{*}(\infty)=P_{1}(\infty)+P_{2}(\infty)+\ldots+P_{5}(\infty)=\frac{N_{4}}{D_{2}}
\end{gathered}
$$

$$
\begin{aligned}
& N_{3}=\alpha_{1} \alpha_{2} \alpha_{3}\left(\alpha_{1}+\alpha_{2}+\beta_{2}\right)+\alpha_{2} \alpha_{3} \beta_{1}\left(\alpha_{1}+\alpha_{2}\right) \\
& +\alpha_{1} \alpha_{3} \beta_{2}\left(\alpha_{1}+\alpha_{2}+\beta_{1}+\beta_{2}\right)+\alpha_{2} \alpha_{3} \beta_{1} \beta_{2} \\
& N_{4}=\alpha_{2} \alpha_{3} \beta_{1}\left(\alpha_{1}+\alpha_{2}\right)+\alpha_{1} \alpha_{3} \beta_{2}\left(\alpha_{1}+\alpha_{2}+\beta_{1}+\beta_{2}\right) \\
& +\alpha_{2} \alpha_{3} \beta_{1} \beta_{2}+\alpha_{1} \beta_{2} \beta_{3}\left(\alpha_{1}+\alpha_{2}+\beta_{1}+\beta_{2}\right)+\alpha_{2} \beta_{1} \beta_{2} \beta_{3} \\
& D_{2}=\alpha_{1}^{2} \alpha_{3} \beta_{2}+\alpha_{1} \alpha_{2} \alpha_{3} \beta_{1}+\alpha_{1} \beta_{2}^{2} \beta_{3}+2 \alpha_{1} \alpha_{2} \alpha_{3} \beta_{2} \\
& +\alpha_{1} \alpha_{3} \beta_{1} \beta_{2}+\alpha_{2} \alpha_{3} \beta_{1} \beta_{2}+\alpha_{1}^{2} \alpha_{2} \alpha_{3}+\alpha_{1} \alpha_{2}^{2} \alpha_{3}+\alpha_{1} \alpha_{3} \beta_{2}^{2} \\
& +\alpha_{1} \alpha_{2} \beta_{2} \beta_{3}+\alpha \beta_{1} \beta_{2} \beta_{3}+\alpha_{1}^{2} \beta_{2} \beta_{3}+\alpha_{2}^{2} \alpha_{3} \beta_{1}+\alpha_{2} \beta_{1} \beta_{2} \beta_{3} \\
& \qquad F^{*}=C_{0} A_{v}^{*}(\infty)-C_{1} B_{V}^{*}(\infty)
\end{aligned}
$$

\section{Numerical Illustration}

In this section, we numerically obtained and compare the results for system availability and profit function for the developed models. The objectives here are to analyze graphically the effects of system parameters on availability and profit and make comparison. The following set of parameters values are fixed throughout the simulations for consistency.

$$
\begin{aligned}
& \beta_{1}=0.1, \beta_{2}=0.3, \beta_{3}=0.3, \alpha_{1}=0.5, \alpha_{2}=0.5, \\
& \alpha_{3}=0.5, \mu=0.5, C_{0}=200,000, C_{1}=80,000
\end{aligned}
$$

Figure 2 and Figure 3 show the availability results for the system with and without unit exchange being studied against the repair rate $\alpha_{1}$ and exchange rate $\mu$. It is clear from the figure that it is clear that system with unit exchange has higher availability with respect to $\alpha_{1}$ and $\mu$ as compared with the system without unit exchange. The differences between the availability in the system with unit exchanged and without unit exchange widens as $\alpha_{1}$ and $\mu$ increases. These tend to suggest that system with unit exchange is better than the other systems. 


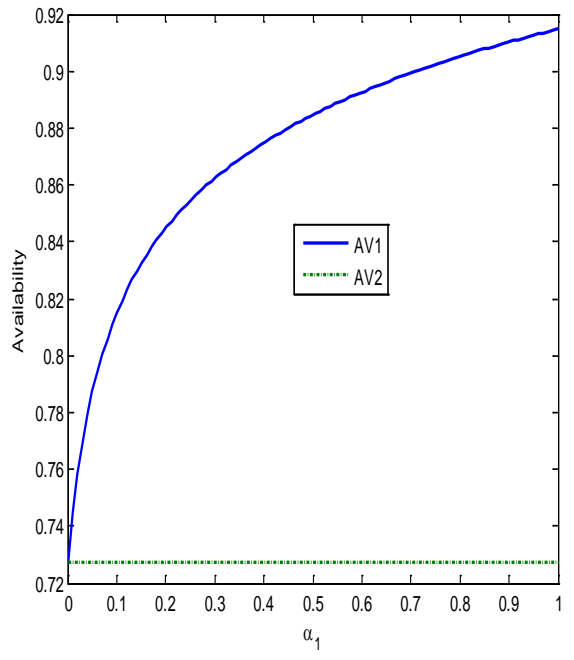

Figure 2. Availability against $\alpha_{1}$

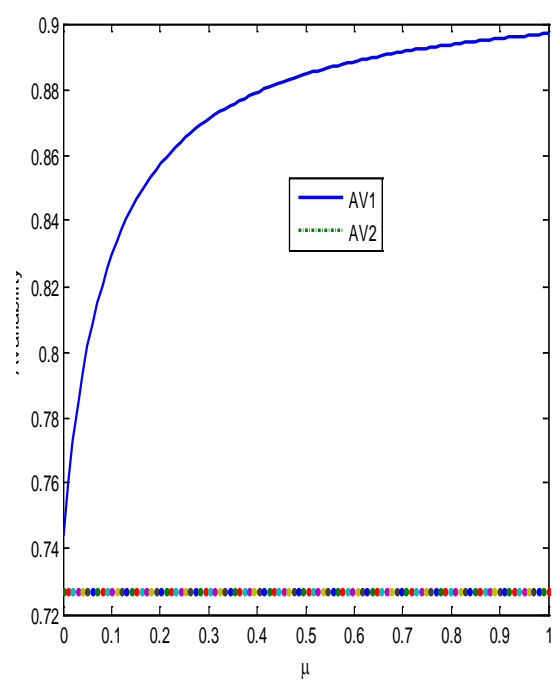

Figure 3. Availability against $\mu$

Figure 4 and Figure 5 depict the profit calculations for the two systems against $\alpha_{1}$ and $\mu$ respectively. The observations that can be made from these figures are much similar to those made on Figure 2 and Figure 3. In these simulations, it can be seen that the profit of the systems increases much slower in Figure 4 than those of Figure 5. We can conclude that system with units' exchange.

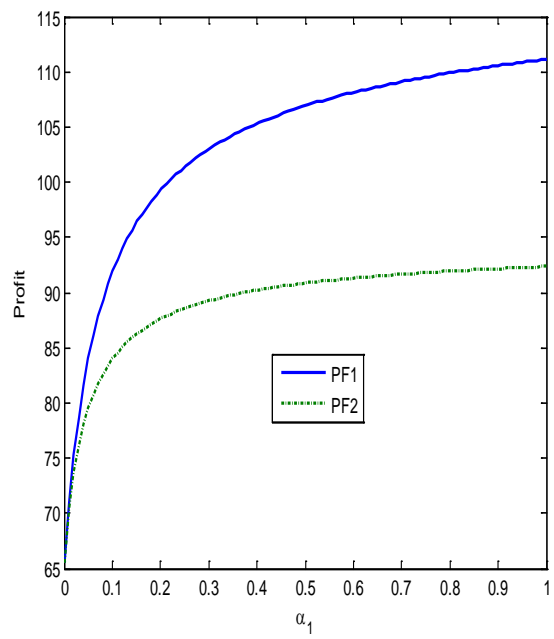

Figure 4. Profit against $\alpha_{1}$

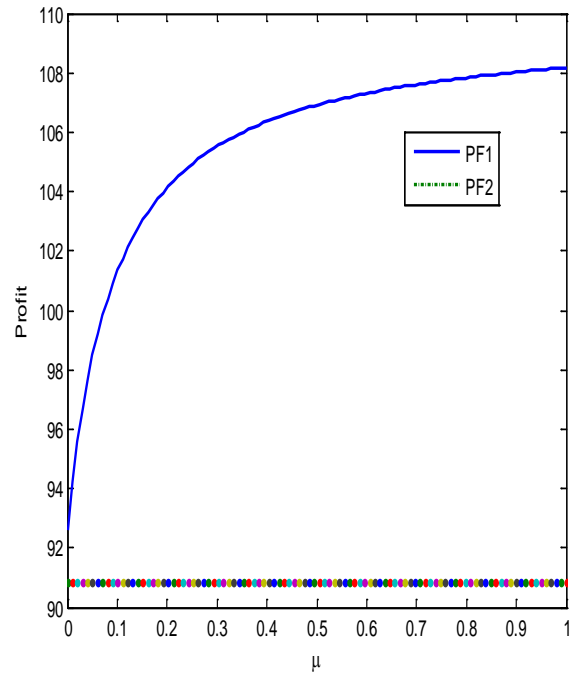

Figure 5. Profit against $\mu$

\section{Conclusion}

In this paper, we constructed a linear consecutive 2-outof-4 repairable system with We have developed the explicit expressions for the availability, busy period and profit function. We perform a parametric investigation of system parameters on MTSF, system availability, busy period and profit function and captured their effect on availability, busy period and profit function. We further performed comparison between system with units exchange and without exchange. From the numerical simulation in Figure 2 - Figure 5, it clear that the entire system with units exchange is better than the system without units' exchange. The present paper show that whenever failed units are exchanged with idle unit, the availability and profit increases. Also the increase in the repair and exchange rate increases the availability and profit.

\section{References}

[1] A. Khatab, N. Nahas, and M. Nourelfath, Availbilty of k-out-of-n: G systems with non identical components subject to repair priorities. Reliab. Eng. Syst. Safety. 94, 2009, 142-151.

[2] I. Yusuf, Availability and profit analysis of 3-out-of-4 repairable system with preventive maintenance, International Journal of Applied Mathematical Research, 1 (4), 2012, pp 510-519.

[3] I. Yusuf, and B. Yusuf, Evaluation of reliability characteristics of two dissimilar network flow systems. Applied Mathematical Sciences, Vol. 7, No. 40, 2013, pp 1983-1999.

[4] I.Yusuf, and N. Hussaini, Evaluation of Reliability and Availability Characteristics of 2-out of -3 Standby System under a Perfect Repair Condition American Journal of Mathematics and Statistics, Vol. 2No. 5, 2012, pp 114-119.

[5] J. Shao, and L.R. Lamberson, Modeling shared-load k-out-of-n : G systems IEEE Trans. Reliab., 40: 1991.

[6] K.M. El-Said, Cost analysis of a system with preventive maintenance by using Kolmogorov's forward equations method, American Journal of Applied Sciences, Vol. 5,No. 4, 2008, pp 405-410.

[7] K.M.El-Said, and M.S. El-Sherbeny, Evaluation of reliability and availability characteristics of two different systems by using linear first order differential equations, Journal of Mathematics, and Statistics, Vol. 1 No. 2, 2005, pp 119-123.

[8] M. Y. Haggag, "Cost analysis of a system involving common cause failure and preventive maintenance, "Journal of Mathematics and Statistics, Vol. 5, No. 4, 2009, pp 305-310. 
[9] Y. Barron, E. Frostig and B.Levikson, Analysis of $r$ out of $n$ systems with several repairmen, exponential life times and phase type repair times: an algorithmic approach. Eur. J. Oper. Res. Algorithm Approach 169, 2006, pp 202-225. 